\title{
The Formation of Galactic Bulges
}

\section{Reynier Peletier*}

Kapteyn Institute, Groningen, The Netherlands

E-mail: peletier@astro.rug.nl

\section{Marc Balcells}

Institute de Astrofísica de Canarias, La Laguna, Spain

E-mail: balcellsell.iac.es

\section{Jesus Falcón-Barroso}

Sterrewacht Leiden, Leiden, The Netherlands

E-mail: jfalconestrw. leidenuniv.nl

\section{Alister Graham}

Mount Stromlo Observatory, Canberra, Australia

E-mail: grahamemso. anu. edu.au

\begin{abstract}
We summarise some recent results about nearby galactic bulges, relevant to their formation. We highlight a number of significant advances in our understanding of the surface brightness profiles, stellar populations, and especially the very centers of spirals. We also view our own Milky Way as if it were an external galaxy. Our main conclusions are that bulges of early-type spirals ( $\mathrm{Sb}$ or earlier) contain central point sources, just like late-type spirals and most other types of galaxies. Central point source luminosities correlate best with total bulge luminosity, and not as well with morphological type. Bulges of early-type spiral galaxies follow the fundamental plane and the colour/line strength vs. luminosity relations of elliptical galaxies. Although we have a reasonable idea about bulges of early-type spirals we know much less about late-type bulges. However, the close resemblance of our Milky Way Bulge to early-type external bulges makes us suspect that maybe bulges of late-type spirals might be very similar as well.
\end{abstract}

Baryons in Dark Matter Halos

5-9 October 2004

Novigrad, Croatia

\footnotetext{
${ }^{*}$ Speaker.
} 


\section{Introduction}

Galactic bulges, objects similar in many ways to elliptical galaxies in the middle of spirals, are the key to study galaxy formation. Although bulges of nearby galaxies have been studied in great detail in recent years (see Kormendy \& Kennicutt 2004 for an in-depth review article), many aspects of them are still unknown. In this short review we will try to show that, contrary to 15 years ago, we are able to make a fairly coherent picture of the nature of galactic bulges, which we can use to test formation models. Rather than trying to comment on all the issues in a much too short space we will concentrate on a few pieces in this puzzle. Before doing his, however, we will first discuss the definition of the bulge. After having talked about surface brightness profiles, stellar populations, and the centers of galaxies, we will end with a short section about our knowledge of bulges at higher redshift.

\section{Definition of Bulges}

One could ask the question whether it makes sense to talk about galactic bulges as separate components of spiral galaxies. If galaxy properties change smoothly from the center outwards, as is e.g. the case for elliptical galaxies, it is not clear whether discussing the inner parts separately makes sense. In the case of bulges one can make the case that they are clearly different from their surrounding disk. They contain less interstellar matter, are generally rounder, have a surface brightness profile that is more strongly peaked, and have different internal kinematics. In the past, Hubble (1936) noted that the normal spiral exhibits a bright, semi-stellar nucleus and a relatively large nuclear region of unresolved nebulosity which closely resembles a lenticular (E7) nebula.

There are a few methods in the literature that are used to calculate the extent of the bulge. The first one is one-dimensional. Here an exponential disk model and an $\mathrm{r}^{1 / 4}$ bulge model are fitted to a major axis surface brightness profile (e.g. Freeman 1970, Kormendy 1977). A generalization of this method takes a Sérsic $\mathrm{r}^{1 / n}$ profile for the bulge (e.g. de Jong 1996). This method can be used for galaxies at all inclinations, but fails when the slope of the surface brightness profiles in bulge and disk region is the same. A different method, using the surface brightness profile together with the morphology of the bulge, was introduced by Kent (1985). Here a galaxy is decomposed into a bulge and a disk of fixed, but different ellipticity, using a non-parametric method. This method uses the fact that bulges are often rounder than their disks, so that in projection the ellipticity in the central regions is lower. This method, only applicable for galaxies that are not face-on, generally gives similar results to the previous one, except in the case of galaxies with boxy bulges (e.g. Jarvis 1986), i.e., edge-on galaxies with rounder, generally very boxy, isophotes. One can think about a third method, using only the stellar kinematics, which decomposes the galaxy into a fast rotating, cold disk and a slowly rotating, hot bulge. This method, which to our knowledge has not been applied to real data yet, might be the most physical, since it separates a galaxy into a slowly rotating elliptical-like spheroid and a fast-rotating disk. Since kinematic analysis has shown that boxy bulges generally are bars seen edge-on (e.g. Bureau \& Freeman 1999), and bars are a phenomenon that occurs in flat disks, the first definition is more in agreement with the third, and for that reason we will use that for the rest of this proceedings.

Apart from decomposing the surface brightness profile in the radial direction it is also of interest to know how the profile in the vertical direction has to be decomposed.we will come to this 


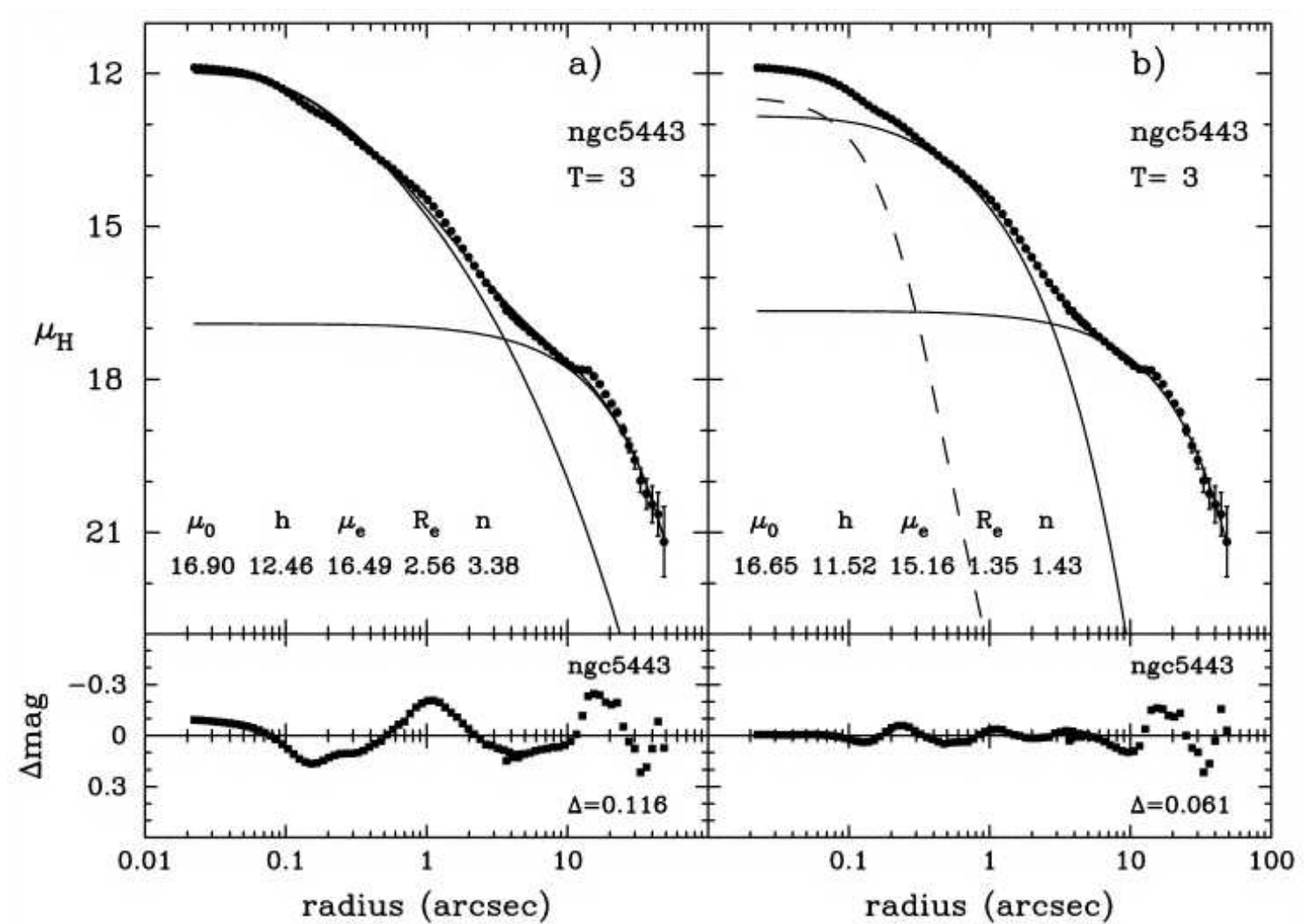

Figure 1: (a) Combined HST plus ground-based H-band surface brightness profile of NGC 5443 (Sb), with PSF-convolved Sérsic bulge and exponential disk components (solid lines). The residuals (data minus model) are shown in the lower panel. (b) Same surface brightness profile fitted with a PSF-convolved Sérsic law, an exponential disk, plus a central point source (dashed line). Residuals are shown in the lower panel. From Balcells et al. 2003.

issue in the next Section.

\section{Surface Brightness Profiles and Components in Bulges}

Inspired by work on elliptical galaxies (Caon et al. 1993) Andredakis et al. (1995) found that surface brightness profiles of bulges are better fit by a Sérsic $r^{1 / n}$ law than by the traditional $r^{1 / 4}$ distribution. With the aid of higher resolution Hubble Space Telescope photometry Balcells et al. (2003) performed the decomposition again on a subsample of the original dataset of Andredakis et al. The results here are quite interesting: in $84 \%$ of the sample a better fit is obtained if a central point source is included. In a moderate fraction of the sample another increase in the goodness of the fit is obtained if an extended component, such as a disk, is included as well. In Fig.1 we show figure 1 of Balcells et al. (2003), which shows the residuals with and without a central point source and an inner disk for an example galaxy.

Given the fact that it is now commonly accepted that large galaxies have central black holes in their centers (e.g. Ferrarese \& Merritt 2000) it is not surprising that bulges contain central point sources, also since many galaxies contain stronger or weaker AGNs. In fact, HST studies of ellipticals and S0 galaxies many central point sources are found (Ravindranath et al. 2001), and also in late-type spirals Carollo et al. (2002) detected nuclear sources in 43 of their 69 objects. Interestingly enough, Balcells et al. also show that the nuclear point source luminosity correlates 


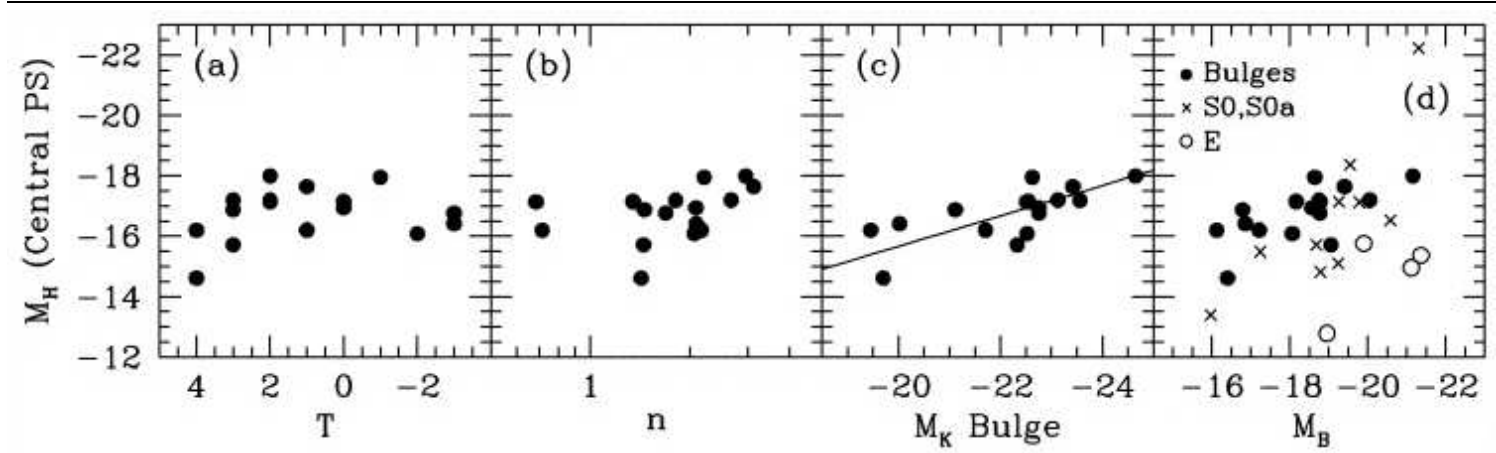

Figure 2: Dependence of the central point source $\mathrm{H}$-band absolute magnitude on global properties of the parent galaxy: (a) $\mathrm{M}_{H, P S}$ vs. galaxy morphological type index T. (b) $\mathrm{M}_{H, P S}$ vs. Sérsic index $n$ of the bulge. (c) $\mathrm{M}_{H, P S}$ vs. $\mathrm{H}-$ band absolute bulge magnitude. The line is an orthogonal regression to the distribution. (d) $\mathrm{M}_{H, P S}$ vs. absolute B-band magnitude. Filled circles: Our bulges; the absolute magnitude is that of the bulge. Crosses: S0 galaxies from Ravindranath et al. (2001). Open circles: E galaxies from Ravindranath et al. (2001). Total galaxy absolute magnitudes are used for S0 and E galaxies. From Balcells et al. 2003

with several quantities, most of all the luminosity of the bulge (Fig.2). The nuclear point sources, containing up to a fraction of order $10^{-3}$ of the bulge luminosity, are easy to accommodate within either a merger origin or a secular evolution origin for bulges. The observed scaling of PS and bulge luminosities does not necessarily indicate an internal origin for the PS since the nuclear gas deposition during a merger may scale with the masses of the merging objects. If bulges are evolved bars, the problem of growing a nuclear star cluster is similar to that of feeding an active galactic nucleus (Maciejewski et al. 2002). If black hole mass correlates with bulge mass, bulge mass correlates with luminosity and nuclear cluster luminosity correlates with black hole mass, this is what one expects.

In Figs. 2 and 3 we also show some other correlations with the luminosity of the central point source. The correlation with the Sérsic index $n$ is strong, while the correlation with morphological type $\mathrm{T}$ is much weaker. In fact, Balcells et al. (2004) show that all correlations with morphological type are less strong than with bulge luminosity. In fact, one of the main conclusions from that paper is that large galaxies are not scale-free, as Courteau et al. (1996) claim, but that they have a scale, namely the luminosity of the bulge. Fig. 3 also shows that the correlation with the central velocity dispersion, and the dust-free colour $I--H$ are not as strong as with bulge luminosity, and that there is no correlation with inclination (disk axis ratio). We would expect a strong correlation with central potential well depth, and possibly the correlation of point source luminosity with velocity dispersion would become stronger if we would make a correction for rotation. We are currently analysing some Integral Field Spectroscopy of this sample to investigate this issue.

Not only from the surface brightness profiles it is apparent that bulges contain other components, such as inner disks, bars, etc., also detailed internal kinematics shows this. For example, the minor axis kinematics of Falcón-Barroso et al. (2003a) shows structures that are different from major axis disks. Moreover, SAURON-data of the S0 galaxy NGC 7332 (Falcón-Barroso et al. 2004) shows that this galaxy most likely has a central disk, a central bar, and a very small kinematically decoupled stellar disk. The gas kinematics in this galaxy is completely decoupled from the stellar kinematics, strongly indicating interactions with the neighbouring galaxy NGC 7339. 


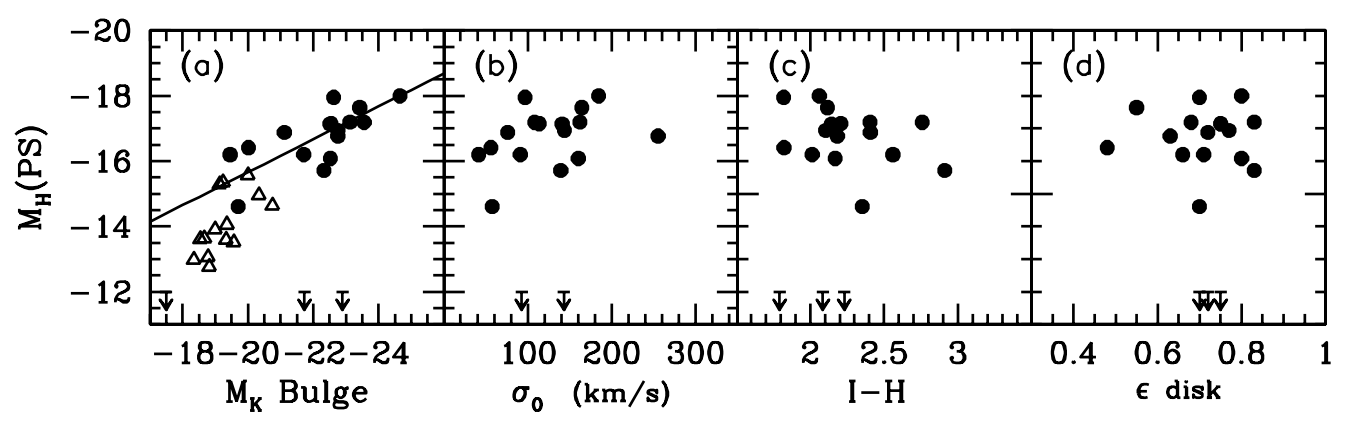

Figure 3: Absolute $H$-band magnitude of the unresolved nuclear sources as a function of: $(a)$ the $K$-band absolute magnitude of the bulge. Filled circles: bulges. Triangles: dwarf ellipticals from Graham \& Guzmán (2003). The line is the regression to the bulge data $(b)$ aperture-corrected central velocity dispersion. (c) Central $I--H$ color, from HST/NICMOS F160W and WFPC2 F814W images (Peletier et al. 1999). (d) Ellipticity of the outer disk, from two-dimensional bulge-disk decomposition in $K$-band images (Andredakis et al. 1995). Upper limits are given for the three galaxies without detected point sources; one of these does not have a central velocity dispersion measurement. From Balcells et al. 2004.

\section{Stellar Populations of Bulges}

At the moment we know relatively little about the stellar populations of bulges, and most of this information is limited to bulges of early-type spiral galaxies. This has to do with the fact that many bulges are dusty, making the interpretation of broadband colours difficult, and that one has to separate a bulge from an often dominating disk. For late-type bulges (types Sbc and later) most of our information comes from studies from HST (e.g. Böker et al. 2002, 2003; Carollo et al. 2002). We know that late-type spirals have nuclear clusters that are more prominent than the ones in early-type spirals. Colours indicate that the central regions are regions full of dust and young stars. Since however, as a result of the fact that the bulge is very small compared to the disk, it is hard to establish whether these young stars and this dust is situated in the disk or in the bulge, we think that it is too early to make definitive statement about bulge populations. In the bulge of our own Milky Way one is able to de-contaminate the bulge from the disk, using e.g. proper motions. In this way Zoccali et al. (2003) find that the age of the Galactic Bulge is old, around $12-13$ Gyr.

For early-type bulges a few significant results have been reported. Broadband colours indicate that bulges are old (10 Gyr with a range of about $2 \mathrm{Gyr}$ ), except for the central regions (Peletier et al. 1999). The position of bulges on the fundamental plane of elliptical galaxies is consistent with this statement. Their formation epoch must have been at most $2.5 \mathrm{Gyr}$ later than ellipticals and S0 galaxies in clusters (Falcón-Barroso et al. 2002). The situation with absorption line strengths is less clear. Falcón-Barroso et al. (2003b) find that the $\mathrm{CaT}^{*}-\sigma$ relation for bulges is the same, with less scatter, as for elliptical galaxies, and decreasing, i.e., the $\mathrm{CaT}^{*}$ index decreases with increasing velocity dispersion, contrary to e.g. the $\mathrm{Mg}_{2}$ index, which is larger with increasing $\sigma$. It is not clear yet what the reason is for this decrease. Possibilities are a Ca-under-abundance w.r.t. Fe, a bottom-heavy IMF, or uncertainties in our knowledge of element-enrichment in galaxies (see also Cenarro et al. 2003). The fact that the relation for ellipticals is the same as for spirals indicates that dust extinction cannot play a major role. The $\mathrm{Mg}_{2}-\sigma$ relation also shows that bulges of early- 


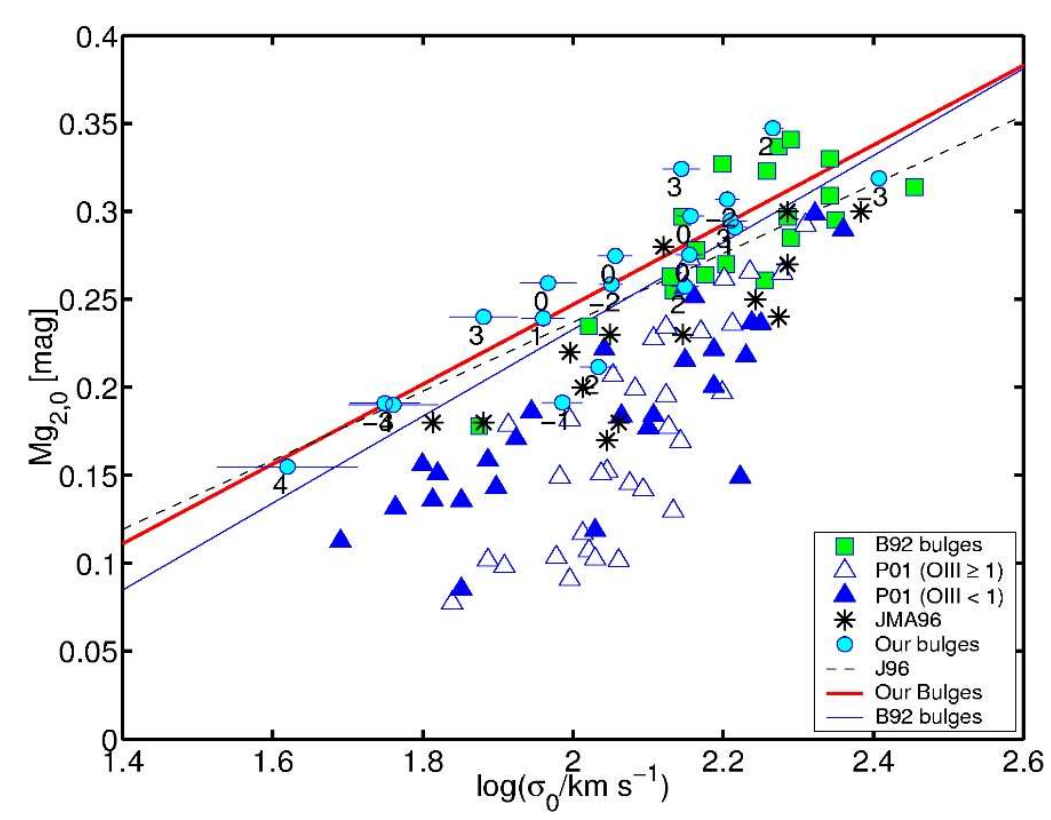

Figure 4: $\mathrm{The}_{\mathrm{Mg}_{2}}-\sigma$ Relation. Filled circles correspond to the sample of bulges of Falcón-Barroso et al. (2002). while solid squares represent the sample of bulges of Bender et al. (1992). Morphological types of the galaxies of our sample are indicated. Open triangles are bulges with $\mathrm{EW}(\mathrm{OIII}) \geq 1$, while filled triangles are bulges with $\mathrm{EW}(\mathrm{OIII})<1$ both from Prugniel et al. 2001. Asteriscs correspond to the sample of bulges of Jablonka et al. 1996. Error bars of $\log \left(\sigma_{0}\right)$ for our sample are also plotted.From Falcón-Barroso et al. 2002.

type spirals behave similar in a similar way as elliptical galaxies (see Fig.4). However, although the data of Falcón-Barroso et al. agree with e.g Jablonka, Martin \& Arimoto (1996) and Bender, Burstein \& Faber (1992), the agreement here with the dataset of Prugniel, Maubon \& Simien (2001) is not very good. The latter data indicate that in general bulges are much younger than ellipticals. This difference might have been caused by a different selection procedure. In the case of the data of Falcón-Barroso et al. much care was taken to make sure that the amount of disk light contaminating the data was limited. In the case of Prugniel et al., however, bulges were selected at random. As a result, it is very likely that star formation in the disk, on the same line of sight as the bulge light, could affect the points and cause the difference. However, one should also realise here that the colours of bulge and inner disk, at least for early type bulges, are generally very similar ( Terndrup et al. (1994), Peletier \& Balcells (1996)) and indicating that the central regions are dominated by old stellar populations. Better quality data will have to settle this issue.

\section{The Centers of Spiral Galaxies}

In the previous discussion we have not talked about the very central regions of spiral galaxies, apart from the central point sources. In Fig.5 we have reproduced Fig, 3a of Peletier et al. (1999). It is clear from this figure is that the filled points for most galaxies are situated far above the grid, 
indicating that they contain dust: the central $\mathrm{A}_{V}$ lies between 0.6 and $1 \mathrm{mag}$. Very few galaxies do not have such a dusty core. As can be seen clearly in the color map of NGC 5838, this dust is often associated with central star formation. So, although most of the bulge is old, some stars are currently formed in the very central regions. In elliptical galaxies a similar phenomenon is seen, since at least $80 \%$ of them contain dust in their central regions (van Dokkum \& Franx 1995). When one looks at absorption line strengths, one also often sees that the innermost point is younger than the rest of the galaxy (e.g. Davies et al. 2001, Sánchez-Blázquez et al. 2004). This is also seen in long slit spectroscopic studies of bulges (Jablonka et al. 2002). Since the material in the center is generally metal rich, the origin of the gas is probably internal, and not gas infalling from outside.

Drawing the analogy with our Galaxy (type $\mathrm{Sbc}$ ), we see a similar structure. Launhardt et al. (2002), using DIRBE data, find an almost exponential bulge, with an enhancement in the region of the Galactic Plane. In that region, with a diameter of $250 \mathrm{pc}$, comparable to the nuclear regions in other galaxies, most of the light comes from cool stars, with a torus of interstellar matter in the same region. Outside this torus there is an absolute lack of interstellar matter. In the very inner parts $(\mathrm{r}<1 \mathrm{pc})$ the light is dominates by hot, massive stars. This shows that the Milky Way bulge is similar in almost all aspects to external bulges of galaxies. It shows that even in an Sbc bulge the the large majority of stars in the bulge is old. Careful studies of other late-type spirals will have to be made to see whether the same is the case there.

\section{Bulges at High Redshift}

Fig.6 is a diagram from Ellis et al. (2001) showing the colour evolution as a function of redshift for galaxies in the Hubble Deep Field. Since here the open symbols are bulges and the filled symbols ellipticals, one would think that at a given redshift the bulges are bluer, and therefore younger ore more metal poor. This conclusion, unfortunately, cannot be drawn from this diagram alone. Bulges are small (bulges that dominate the disk across a radial range of more than $1 \mathrm{kpc}$ are rare), so even in the HDF their size is small. We will have to wait for studies that take into account the contribution from the disk, when studying the bulge. These studies might come soon, i.e. from studies like GOODS, and will lead to interesting progress in the field.

Although we haven't discussed the formation of bulges themselves here, all the results presented here are relevant to obtain the right idea about how spirals formed. For this topic we refer to the review of Kormendy \& Kennicutt (2004) and references in there.

\section{References}

[1] Andredakis, Y. C., Peletier, R. F., \& Balcells, M. 1995, MNRAS, 275, 874

[2] Balcells, M., Graham, A. W., Domínguez-Palmero, L., \& Peletier, R. F. 2003, ApJl, 582, L79

[3] Balcells, M., Graham, A. \& Peletier, R.F., 2004, astro-ph/0404379

[4] Bender, R., Burstein, D., \& Faber, S. M. 1992, ApJ, 399, 462

[5] Böker, T., Stanek, R., \& van der Marel, R. P. 2003, AJ, 125, 1073

[6] Böker, T., Laine, S., van der Marel, R. P., Sarzi, M., Rix, H., Ho, L. C., \& Shields, J. C. 2002, AJ, 123, 1389

[7] Bureau, M., \& Freeman, K. C. 1999, AJ, 118, 126 


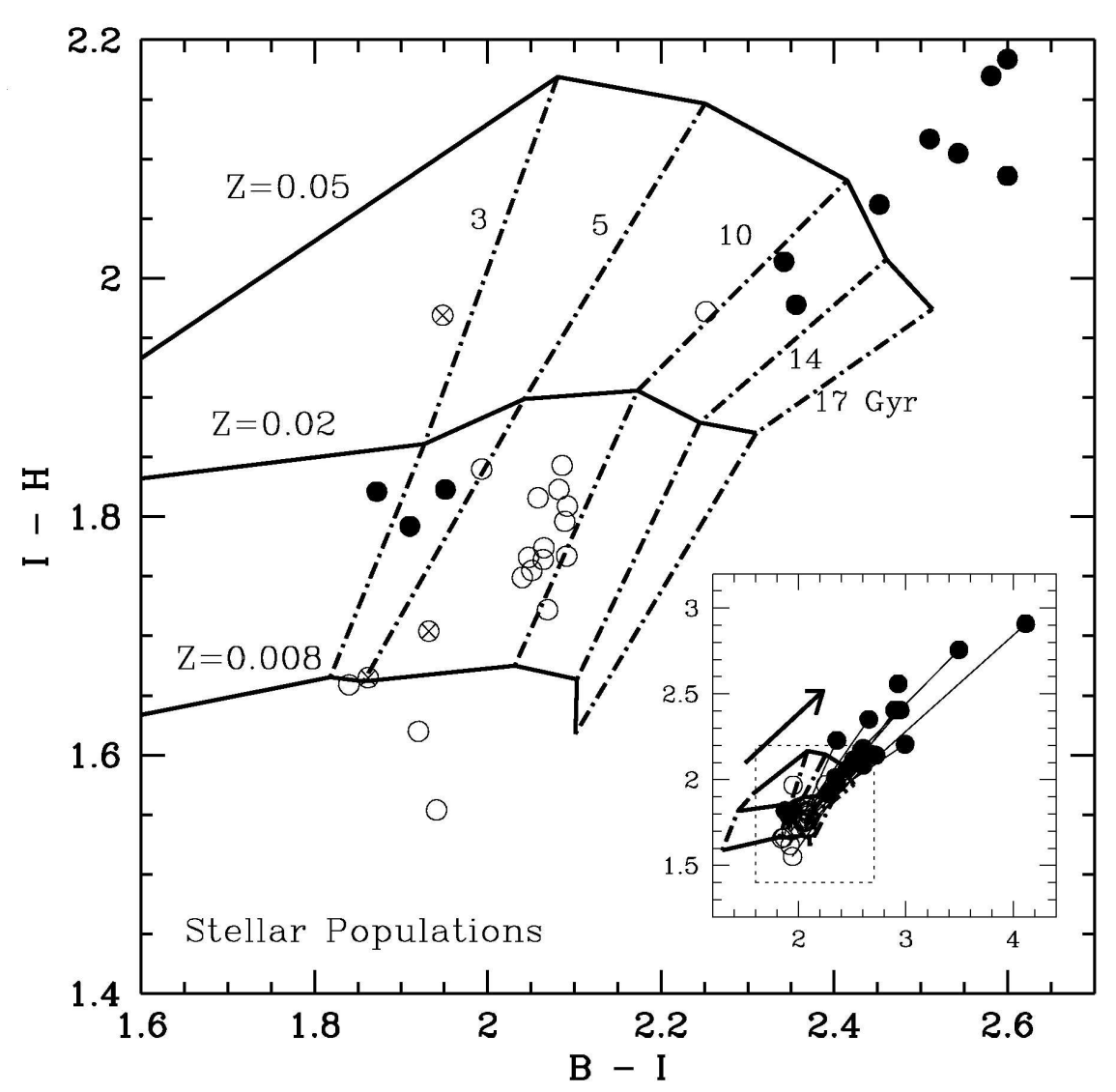

Figure 5: Colour-colour diagrams for the 20 galaxies (from Peletier et al. 1999). Displayed are the positions of the center (filled) and at 1 bulge effective radius on the minor axis (open circles). Reddening vector for a reddening of $A_{V}=1 \mathrm{mag}$ (inset) are given as well. Sbc galaxies are indicated with circled crosses. Superimposed are SSP models of Vazdekis et al. (1996). Solid lines are lines of constant metallicity, dashed-dotted lines are loci of constant age.

[8] Caon, N., Capaccioli, M., \& D’Onofrio, M. 1993, MNRAS, 265, 1013

[9] Carollo, C. M., Stiavelli, M., Seigar, M., de Zeeuw, P. T., \& Dejonghe, H. 2002, AJ, 123, 159

[10] Cenarro, A. J., Gorgas, J., Vazdekis, A., Cardiel, N., \& Peletier, R. F. 2003, MNRAS, 339, L12

[11] Courteau, S., de Jong, R. S., \& Broeils, A. H. 1996, ApJl, 457, L73

[12] Davies, R. L., et al. 2001, ApJl, 548, L33

[13] de Jong, R. S. 1996, A\&AS, 118, 557

[14] Ellis, R. S., Abraham, R. G., \& Dickinson, M. 2001, ApJ, 551, 111

[15] Falcón-Barroso, J., Balcells, M., Peletier, R. F., \& Vazdekis, A. 2003, A\&A, 405, 455

[16] Falcón-Barroso, J., Peletier, R. F., Vazdekis, A. \& Balcells, M., 2003, ApJ, 588, L17

[17] Falcón-Barroso, J., et al. 2004, MNRAS, 350, 35

[18] Ferrarese, L., \& Merritt, D. 2000, ApJl, 539, L9

[Fre70] Freeman, K. C. 1970, ApJ, 160, 811 


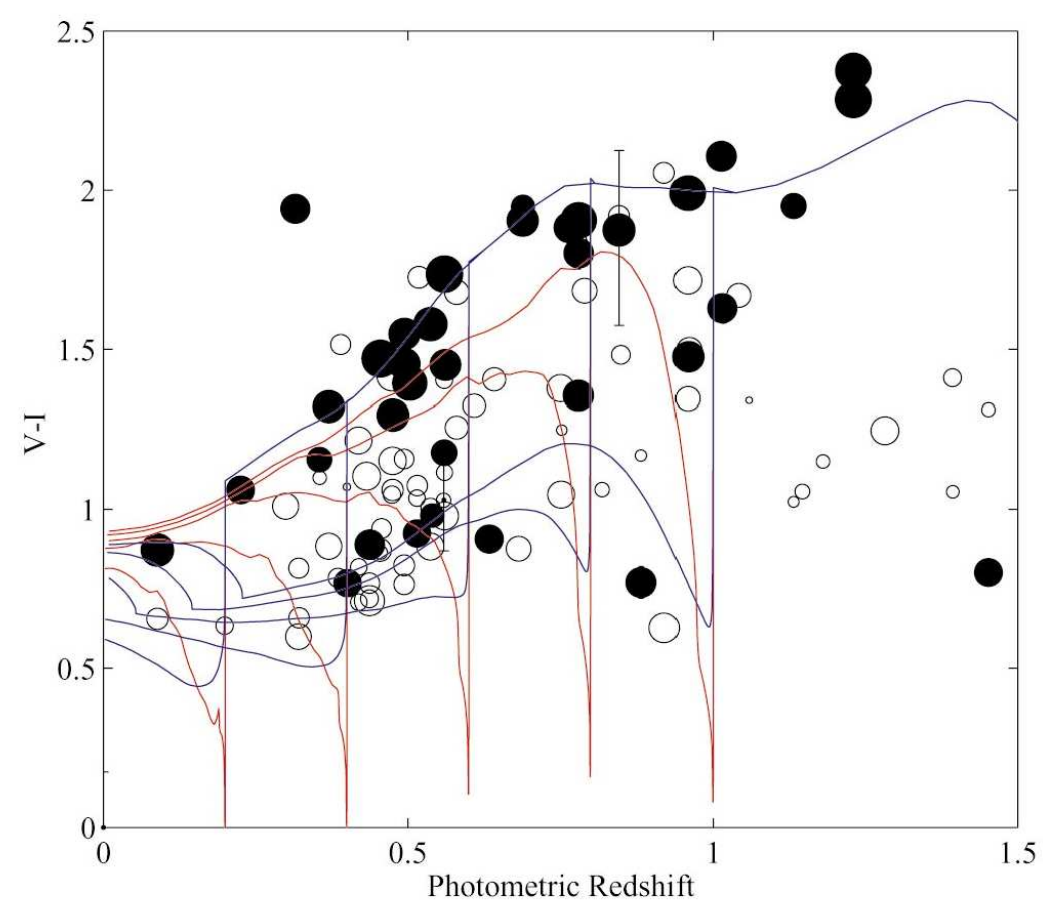

Figure 6: V-I colors for ellipticals (solid symbols, large aperture) and spiral bulges (open symbols, 5\% aperture ) as a function of photometric redshift. Symbol sizes are proportional to galactic central concentration. The solid blue curve at the top represents the observed V-I color expected for a passively evolving system that formed in a single burst at $\mathrm{z}=3$. This is the baseline model upon which secondary bursts are added. The other curves define color-redshift trajectories for systems that suffer secondary bursts of activity at redshifts of $0.2,0.4,0.6,0.8$, and 1 . Red curves refer to 0.1 Gyr bursts involving $15 \%$ of the stellar mass, whereas the blue curves refer to extended bursts with 5 Gyr e-folding time scales (from Ellis et al. 2001).

[19] Graham, A. W., \& Guzmán, R. 2003, AJ, 125, 2936

[20] Hubble, E. P. 1936, Yale University Press,

[21] Jablonka, P., Martin, P., \& Arimoto, N. 1996, AJ, 112, 1415

[22] Jablonka, P., Gorgas, J., \& Goudfrooij, P. 2002, ApSS, 281, 367

[23] Jarvis, B. J. 1986, AJ, 91, 65

[24] Kent, S. M. 1985, ApJs, 59, 115

[25] Kormendy, J. 1977, ApJ, 218, 333

[26] Kormendy, J., \& Kennicutt, R. C. 2004, ARAA, 42, 603

[27] Launhardt, R., Zylka, R., \& Mezger, P. G. 2002, A\&A, 384, 112

[28] Maciejewski, W. 2002, Astronomical Society of the Pacific Conference Series, 275, 251

[29] Peletier, R. F., \& Balcells, M. 1996, AJ, 111, 2238

[30] Peletier, R. F., \& Balcells, M. 1997, New Astronomy, 1, 349

[31] Peletier, R. F., Balcells, M., Davies, R. L., Andredakis, Y., Vazdekis, A., Burkert, A., \& Prada, F. 1999, MNRAS, 310, 703 
[32] Prugniel, P., Maubon, G., \& Simien, F. 2001, A\&A, 366, 68

[33] Ravindranath, S., Ho, L. C., Peng, C. Y., Filippenko, A. V., \& Sargent, W. L. W. 2001, AJ, 122, 653

[34] Sánchez-Blázquez, P., 2004, Ph.D. Thesis, Universidad Complutense Madrid

[35] Terndrup, D. M., Davies, R. L., Frogel, J. A., Depoy, D. L., \& Wells, L. A. 1994, ApJ, 432, 518

[36] van Dokkum, P. G., \& Franx, M. 1995, AJ, 110, 2027

[37] Vazdekis, A., Casuso, E., Peletier, R. F., \& Beckman, J. E. 1996, ApJs, 106, 307

[38] Zoccali, M., et al. 2003, A\&A, 399, 931 